\title{
Seroprevalence and associated risk factors of mosquito-borne alphaviruses in horses in northern Queensland
}

\author{
B Gummow, ${ }^{\mathrm{a}, \mathrm{b} *}$ RHH Tan, ${ }^{\mathrm{a} *}$ RK Joice, ${ }^{\mathrm{a}}$ G Burgess ${ }^{\mathrm{a}}$ and J Picard ${ }^{\mathrm{a}}$
}

\begin{abstract}
Objective To investigate the seroprevalence and associated risk factors of alphaviruses (Ross River virus (RRV), Barmah Forest virus (BFV) and Whataroa virus (WHAV)) in northern Queensland horses.

Methods A cross-sectional study of alphavirus antibodies in horses $(n=287)$ from 147 properties in northern Queensland from September 2013 to June 2014 was conducted. Owners of sampled horses were interviewed on potential risk factors. Data were analysed for associations using multivariable logistic regression.
\end{abstract}

Results Antibody titres for RRV were demonstrated in samples from 134 properties (91\%; 95\% confidence interval (Cl) 87-96\%); 22 properties (15\%) had BFV reactors (95\% Cl 12-18\%) and 2 properties $(1.4 \%)$ had WHAV reactors $(95 \% \mathrm{Cl}-0.5-3.2 \%)$. The highest seroprevalence of RRV was in the Townsville-Burdekin region (93\%; 95\% Cl 90-96\%) followed by the Mackay-Whitsunday (90\%; 95\% Cl 88-98\%) and Far North Coast-Tableland (82\%; 95\% Cl 74-90\%) regions. No association ( $\mathrm{P} \leq 0.05$ ) could be shown between any of the viruses and age groups, sexes, annual average temperature, degree of rainfall or proximity to wet environments. An association with reported large numbers of mosquitoes was seen for RRV but not BFV. A significant association between properties in close proximity to poultry and pigs was shown for BFV.

Conclusion RRV is endemic within the horse population of northern Queensland, but horses exhibit few clinical signs and could play a role as amplifying hosts in the tropics. Exposure of horses to BFV is significant in northern Queensland and it should be considered a differential diagnosis for RRV. WHAV warrants further study.

Keywords Barmah Forest virus; horses; northern Queensland; risk factors; Ross River virus; seroprevalence; Whataroa virus

Abbreviations AP, apparent prevalence; BFV, Barmah Forest virus; $\mathrm{Cl}$, confidence interval; df, degrees of freedom; rpm, revolutions per minute; RRV, Ross River virus; WHAV, Whataroa virus

Alphaviruses are one of the two genera belonging to the Togaviridae family of viruses that have been associated with severe polyarthralgia and neurological disease, including encephalitis, in humans and equids. Australia has several zoonotic

${ }^{*}$ Corresponding authors.

${ }^{a}$ College of Public Health, Medical and Veterinary Sciences, James Cook University, Townsville, Queensland, Australia; rachel.tan@jcu.edu.au; bruce.gummow@jcu.edu.au ${ }^{b}$ Department of Production Animal Studies, Faculty of Veterinary Science, University of Pretoria, Pretoria, South Africa mosquito-borne viruses, with two of these viruses being Ross River virus (RRV) and Barmah Forest virus (BFV).

RRV is endemic in Australia and is the most common vector-borne disease in humans, accounting for $56 \%$ of the total notifications in an annual report of the National Notifiable Diseases Surveillance System. ${ }^{1}$ Infection by RRV in horses is also significant, with seroprevalence reported at $56 \%$ in one study of Victorian horses suspected to have contracted the disease and in $65 \%$ of horses involved in a serological survey in New South Wales. ${ }^{2,3}$

BFV is currently the second most frequently reported mosquito-borne disease of humans in Australia, at $21 \%$ of total notifications. ${ }^{1}$ A striking feature of human disease in 2006-07, which had the second highest number of notifications on record, was the unusually high and significant number of notifications outside peak seasonal months. ${ }^{4}$ This could have been indicative of changing favourable climatic conditions allowing increased breeding of vectors. ${ }^{4} \mathrm{BFV}$ induces polyarthritis-like illness in humans with symptoms similar to those of RRV. ${ }^{5}$ Unlike RRV, the hosts and vectors of BFV are not as evident or as well understood. Seropositivity has been identified in several different non-human species, including possums, dogs, cats and horses. ${ }^{6}$ Although there is no conclusive evidence that BFV may cause clinical disease in horses, it is possible that it may cause signs similar to RRV.

Whataroa virus (WHAV) is an alphavirus belonging to the Western equine encephalitis serological group. The virus is the only known indigenous mosquito-borne virus in New Zealand. Antigenic variants of the New Zealand strain of WHAV have been isolated from mosquitoes captured in New South Wales, but there is currently no conclusive evidence that it causes clinical disease in humans or animals. ${ }^{7}$

There are no current data on the seroprevalence of alphaviruses in horses in northern Queensland, yet Queensland has the highest number of human RRV notifications. ${ }^{1}$ This study aimed to provide important information on exposure of horses in northern Queensland to a selection of alphaviruses and examine the risk factors that may be associated with these viruses.

\section{Materials and methods}

\section{Survey and study area}

Ethics approval was obtained from James Cook University Human and Animal Ethics Committees (approval nos. H5536 and A1997, respectively) to carry out a cross-sectional study, commencing September 2013 through June 2014, of the domestic horse population of northern Queensland (regions north of Rockhampton and Longreach). Serum samples were collected from horses of consenting owners participating in regional equestrian events and from horses admitted to regional equine veterinary practices. The majority of 
samples were collected at participating equestrian events. The study excluded horses that were severely ill or had a history of chronic infections or underlying immunosuppressive disease. The exclusion criteria included horses that were presented for emergency evaluation or surgery, had clinical evidence of severe systemic metabolic or cardiovascular disturbance, an infectious disease process, lymphopenia and/or neutropenia or had been diagnosed with an immunemediated condition. Horses that had not been in the study area for at least 6 months prior to the time of blood collection were also excluded from sampling.

The number of horses to be included in the survey was based on the sensitivity (99\%) and specificity (84\%) of the serum microneutralisation test, ${ }^{8}$ an assumed prevalence of $90 \%$ RRV-seropositive horses ${ }^{2}$ and an allowable error of $5 \%$ using a confidence level of $95 \%$. Using these assumptions, a sample size of 189 horses was calculated for the prevalence survey. ${ }^{9,10}$ The assumed prevalence of $90 \%$ for RRV was based on a pilot study of 10 horses tested at James Cook University prior to the survey. The pilot horses were used for teaching purposes and had been resident in northern Queensland for several years.

\section{Sample collection and storage}

A veterinarian collected a $10-\mathrm{mL}$ red stopper (plain glass) and a 10 -mL green stopper (EDTA) vacutainer blood sample from the jugular vein of each horse using a 19-gauge needle. The red stopper samples were stored at $+4^{\circ} \mathrm{C}$ overnight and then centrifuged at $+20^{\circ} \mathrm{C}$ for $15 \mathrm{~min}$ at $18,000 \mathrm{rpm}$. Serum from the red stopper tubes was aliquoted into sterile cryovials and stored at $-20^{\circ} \mathrm{C}$ until analysis. The EDTA plasma samples were not used in this study and will not be discussed further.

\section{Questionnaire survey}

A structured questionnaire survey was completed by each horse owner at the time of blood collection or via a telephone interview thereafter. The questionnaire covered the previous 12-month period of the horse and comprised 32 questions designed to acquire information on the horse's demographic profile (age, sex, breed), associated geographic and environmental factors (location of the property on which the horse was housed in reference to coastal areas, marshlands, waterways and wetland and the type of agricultural activities prevalent in the area), management factors (water sources, housing conditions, other animal species present in the horse paddock and in adjoining paddocks) and observance of clinical signs that might be associated with viral infection (ataxia, submandibular lymphadenopathy, distal limb and/or ventral abdominal oedema, muscle stiffness, pyrexia, abortion, still births). Information regarding the annual average rainfall and temperature corresponding to each property was also obtained from the Australian Bureau of Meteorology (Climate statistics for Australian sites, Commonwealth of Australia 2014; http://www.bom.gov.au/).

\section{Sample analysis}

Serum samples were tested at James Cook University, Townsville, for the presence of antibodies to RRV T48, BFV K5540 and WAV M78 using the microneutralisation assay method. The Reed and Muench tissue culture infectious dose 50 calculation method was used to establish the working dilution of viral antigen for each of the three viruses. ${ }^{11}$ Each serum sample was transferred in batches of 80 to an
$8 \times 12$-well clear polypropylene plate with a non-treated surface, labelled accordingly and frozen at $-20^{\circ} \mathrm{C}$ until required.

Sera samples were serially diluted in a 96-well flat-bottom microtitre plate (Sarstedt, Germany) using Dulbecco's modified eagle medium (DMEM with 5\% fetal bovine serum [FBS]) to give final dilutions between $1: 10$ and $1: 1280$.

DMEM with 5\% FBS was added to column 12 to act as a cell control while column 11 was the viral control. Plates were covered and incubated for $1 \mathrm{~h}$ at $37^{\circ} \mathrm{C}$ in a $5 \% \mathrm{CO}_{2}$ atmosphere. Aliquots of $50 \mu \mathrm{L}$ containing a suspension of 20,000 Vero cells $\left(4 \times 10^{5}\right.$ cells $\left./ \mathrm{mL}\right)$ in DMEM with $5 \%$ FBS was added to all wells of the plates, which were then incubated at $37^{\circ} \mathrm{C}$ in a $5 \% \mathrm{CO}_{2}$ atmosphere and monitored daily for 6 days when cytopathic effect was recorded and serum antibody titres were calculated. This process was repeated for each virus until all serum samples had been tested for all three viruses.

\section{Data analysis}

Apparent prevalence (AP) and its corresponding 95\% confidence interval (CI) of each virus were calculated using proportions of properties testing positive to the specific virus. Any property with at least one horse reacting positively to the virus at $1: 10$ dilution was assumed to be seropositive. Similarly, the AP of each virus on horse properties located within the Far North Coast-Tableland region, the TownsvilleBurdekin region and the Mackay-Whitsunday region, together with their 95\% CIs, were also determined and compared. The CIs for AP were calculated using formulae adopted from Cameron. ${ }^{12}$

Survey data were entered into Microsoft Excel (2007) and the computer software program NCSS (NCSS Inc., Kaysville, UT, USA) for analysis. The associations between seropositive horses/properties and various risk factors were analysed using Chi-square tests (i.e. univariate analysis). Risk factors with a P-value $<0.20$ on univariate analysis were selected to be included in the multivariable logistic regression models. ${ }^{13} \mathrm{~A}$ hierarchical stepwise forward elimination process with switching of variables based on log likelihood values was used to determine the best fitting logistic regression model. NCSS has a built-in algorithm that does the selection process and several iterations were run before the final model was selected, which contained the best fitting log likelihood value and only the variables with a $\mathrm{P}$-value $<0.05$ on the Wald test. ${ }^{14,15}$

\section{Results}

Over the 10-month period of the study (September 2013 to June 2014), 287 horse and one donkey serum samples were collected from 147 properties in northern Queensland (Figure 1). Age ranged from 1 to 33 years, with 160 males and 127 females.

\section{Ross River virus}

Distribution of the microneutralisation assay titres for RRV ranged from 10 to 1280 , with the most frequently recorded titre being 80 (29\% of serum samples, Figure 2). Of the 287 horses, 263 showed exposure to RRV at serial two-fold dilutions (1:10 to $1: 1280)$ of serum, correlating to a seropositivity of $91 \%$ (95\% CI $88-95 \%)$. The samples originated from 147 properties across northern Queensland and 134 (91\%) of these were found to have horses that had been 


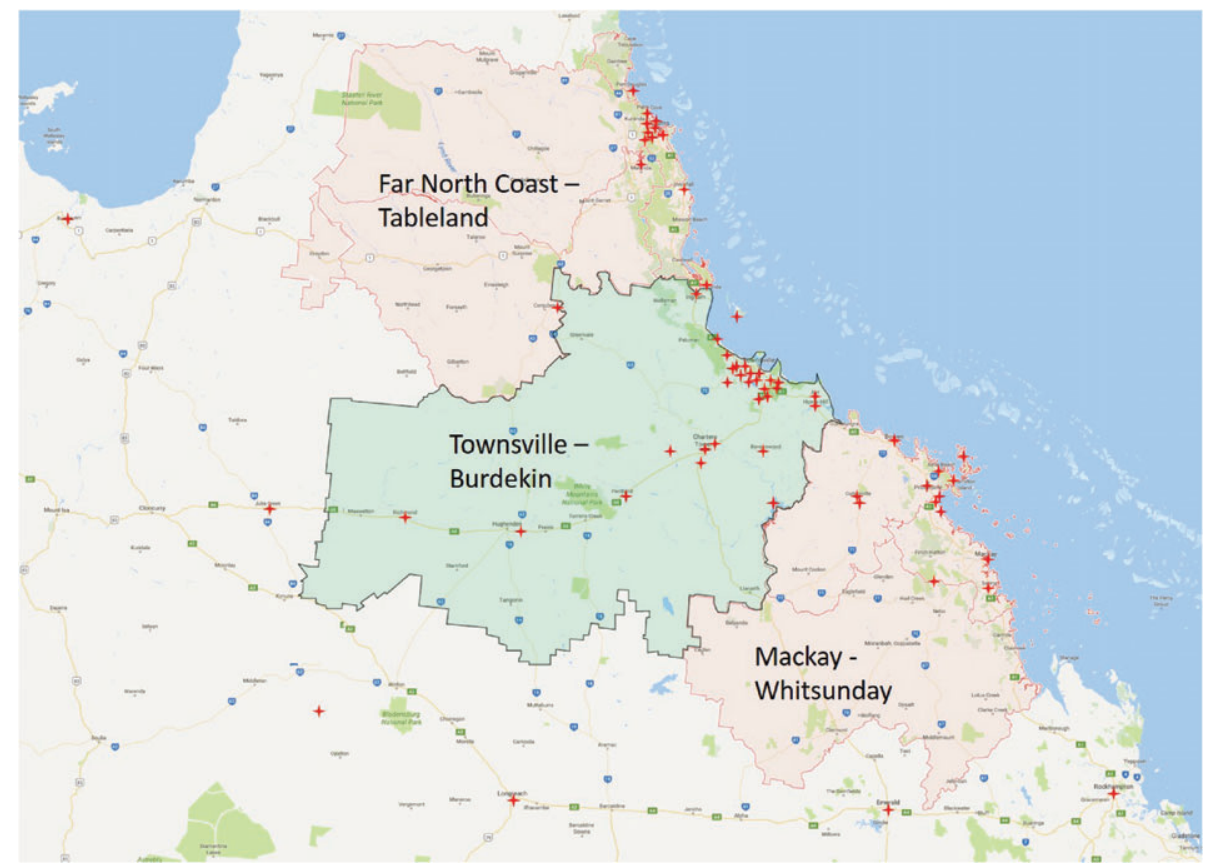

Figure 1. Distribution of properties (+) in northern Queensland sampled for the pres-ence of Ross River virus, Barmah Forest virus and Whataroa virus, with regional divisions indicated.

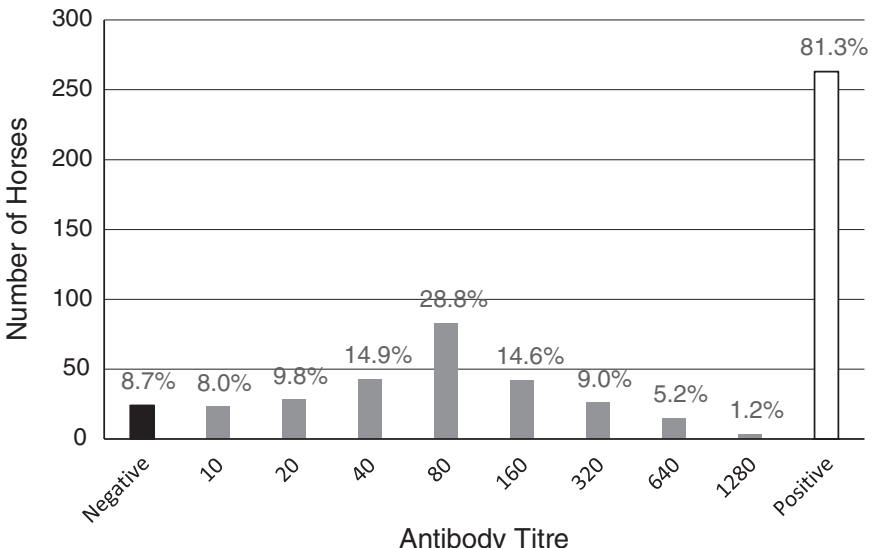

Figure 2. Distribution of microneutralisation assay titres for Ross River virus antibodies in horses in northern Queensland, with number of horses and percentages for each titre.

exposed to the virus (Figure 3). Adjusting for clustering among properties, ${ }^{10}$ the true prevalence of RRV antibodies in horses within the areas surveyed was found to be between $87 \%$ and $96 \%$.

From the Far North Coast-Tableland region, 38 horses residing on 28 properties were sampled, of which 31 from 23 properties were found to be seropositive to RRV. In the Townsville-Burdekin region, 201 horses residing on 89 properties were sampled and 190 from 83 properties were found to be seropositive, while in the MackayWhitsunday region 49 horses residing on 30 properties were sampled and 42 from 28 properties were seropositive. The proportion of seropositive properties in the Far North Coast-Tableland, the Townsville-Burdekin region and the Mackay-Whitsunday regions was $82 \%$ (95\% CI $74-90 \%$ ), 93\% (95\% CI 90-96\%) and 90\% (95\% CI $88-98 \%)$, respectively. No significant difference could be shown between each of the regions sampled $\left(\chi^{2}=3.5, \mathrm{df}=2, \mathrm{P}=0.16\right)$.
The seroprevalence of RRV by demographic, geographic and management factors is presented in Table 1. Seroprevalence of RRV did not differ significantly between the sexes $\left(\chi^{2}=0.10 ; \mathrm{df}=2\right.$; $P=0.95)$. The age of the sampled horses ranged from 1 to 33 years, with a median age of 11 years. No significant association between seropositivity and age group was detected $\left(\chi^{2}=2.94, \mathrm{df}=5\right.$, $P=0.71)$. Horses were also categorised by breed encompassing the highest represented purebred breeds; crossbred animals and the remaining horses not represented with enough numbers to be considered as a category were grouped as 'other'. No significant association between seropositivity and breed was observed $\left(\chi^{2}=4.36\right.$, $\mathrm{df}=5, \mathrm{P}=0.50$ ). However, with a P-value of $<0.2$ for Thoroughbred horses and crossbred horses $(\mathrm{P}=0.17$ and $\mathrm{P}=0.11$ respectively), both were included in the multivariable logistic regression models.

On the horse properties included in the survey the annual average temperature ranged between $19.1^{\circ} \mathrm{C}$ and $25^{\circ} \mathrm{C}$; the median temperature was $24.4^{\circ} \mathrm{C}$. The 147 horse properties were divided into one of two categories according to their annual average temperature $\left(<23^{\circ} \mathrm{C}\right.$ and $\geq 23^{\circ} \mathrm{C}$ ). The horse properties were also divided into one of two categories according to their annual average rainfall $(<1000 \mathrm{~mm}$ and $\geq 1000 \mathrm{~mm}$ ). On the horse properties included in the survey the annual average rainfall received ranged between 393 and $3573 \mathrm{~mm}$. There was no significant association between seropositivity and either annual average temperature (Yates' $\chi^{2}=3.04, \mathrm{df}=1, \mathrm{P}=0.11$ ) or rainfall (Yates' $\chi^{2}=1.01, \mathrm{df}=1, \mathrm{P}=0.17$ ). P-values for both temperature and rainfall were $<0.2$ in the univariate analysis and were therefore included in the multivariable logistic regression models. In order to account for possible collinearity, temperature and rainfall were analysed separately within the models.

Association with seropositivity and horse properties with large numbers of mosquitoes in the previous 12 months was found to be significant $\left(\chi^{2}=4.23 ; \mathrm{df}=1 ; \mathrm{P}=0.03\right)$ in the univariate analysis and this variable was analysed further within the multivariable logistic 


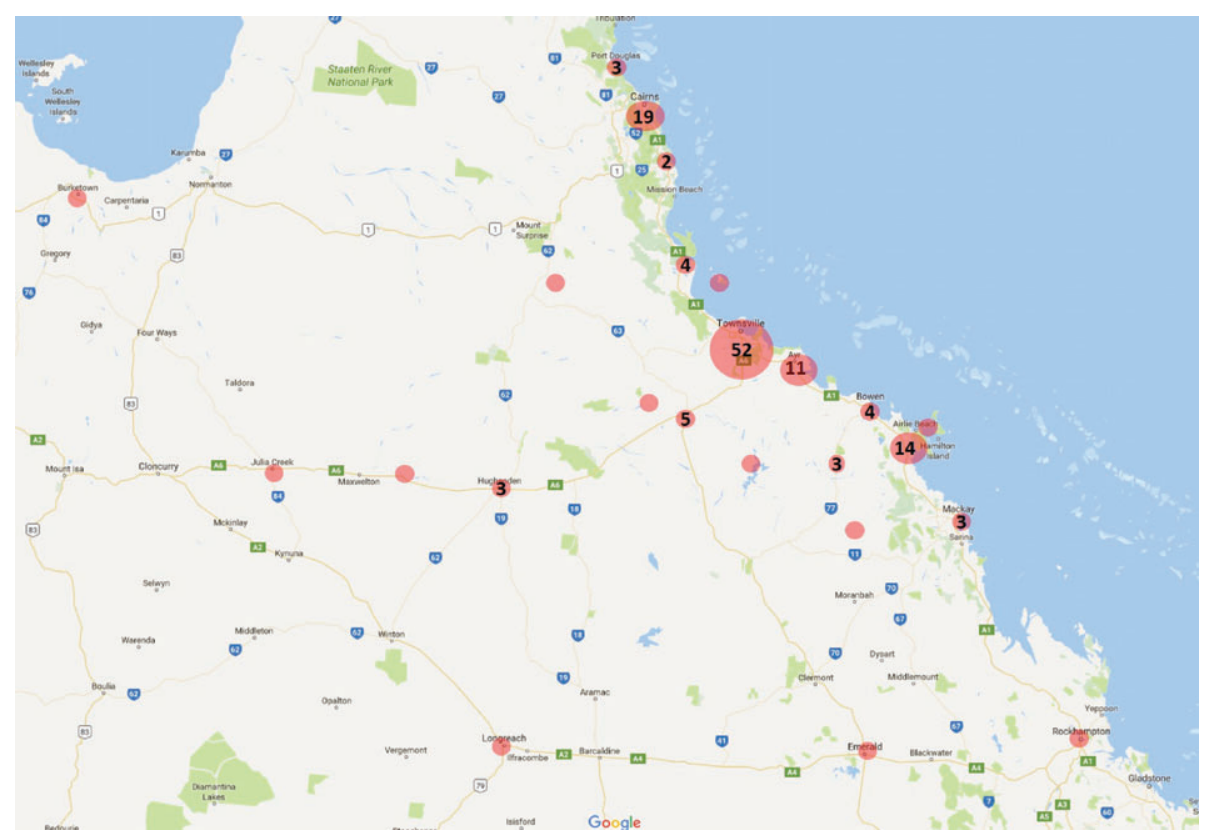

Figure 3. Clustering of properties with Ross River virus seropositive horses in northern Queensland. The shaded circles represent properties on which seropositive horses were housed - single properties represented by unnumbered circles and locations with $>1$ properties having a number identifying the number of properties in that location.

regression models. It was found to be the only remaining risk factor associated with RRV seropositivity $(\mathrm{P}=0.06$, Table 2$)$ if a significance level of $10 \%$ was regarded as acceptable. The presence of other animal species, including avian (domestic and wild), bovine, porcine (domestic and feral), canine (including dingoes) and marsupial, on the property and/or on those within the neighbourhood, and their influence on the status of the property being seropositive to the virus, were also analysed. Avian species had a P-value $<0.2$ in the univariate analysis, but the variable was removed during multivariable logistic regression analysis because of a combination of the small category size and all outcomes being positive, which in turn disrupted the model. Permanent or temporary flying fox roosts on or near the properties were not identified or analysed.

Horse properties were further analysed to determine if the locality of the property in close proximity to waterways, marshlands, coastal regions, wetlands, creeks, rivers and/or dams had an influence on the status of the property being seropositive to RRV. No significant association between seropositivity and proximity to such water sources could be shown (Yates' $\chi^{2}=0.18 ; \mathrm{df}=1 ; \mathrm{P}=0.34$ ).

Within the questionnaire, horse owners were asked if their horses had exhibited signs related to RRV at any stage over the 12 months prior to the animals being tested. Only horses that demonstrated antibodies at a titre $\geq 1: 640$ were involved at this stage of the study. In total, 14 horses were identified with titres $\geq 1: 640$. Analysis of the data on ataxia, stiffness and soreness (both muscular and skeletal), inflammation of joints, swelling of the limbs, high fever, respiratory conditions, episodes of colic, lassitude and periods of abnormal irritability did not show any definitive association of these signs with seropositivity to RRV. Of the 14 horses assessed, four showed at least one of these symptoms. One horse was noted to have experienced inflammation of joints as well as stiffness and muscle tenderness, and another experienced joint stiffness and muscle tenderness only. The third displayed a prolonged period of lassitude and signs of very little energy, and the fourth showed signs of a respiratory condition.

\section{Barmah Forest virus}

A total of 22 horses of the 288 tested, originating from 22 of the 147 properties across northern Queensland, showed evidence of exposure to BFV (seropositivity 7.6\%; 95\% CI 6.00-9.00\%). The total proportion of properties found to be seropositive to the virus was $15 \%$ (95\% CI $12.00-18.00 \%)$.

From the Far North Coast-Tableland region, 38 horses residing on 28 properties were sampled, of which six horses from six different properties showed evidence of exposure to BFV. In the Townsville-Burdekin region 201 horses residing on 89 properties were sampled and 13 horses from 12 properties were found to be seropositive, while in the Mackay-Whitsunday region 49 horses residing on 30 properties were sampled and four horses from four properties were seropositive. The proportion of seropositive properties in the Far North Coast-Tableland, the Townsville-Burdekin region and the Mackay-Whitsunday regions was $21.4 \%$ (95\% CI $13-30 \%$ ), $13.5 \%$ (95\% CI 10-17\%), and $13.3 \%$ (95\% CI 7-20\%), respectively. No significant difference could be shown between each of the regions sampled $\left(\chi^{2}=1.14, \mathrm{df}=2, \mathrm{P}=0.57\right)$. The seroprevalence of BFV by demographic, geographic and management factors is presented in Table 3. Seroprevalence of BFV did not differ significantly between the sexes $\left(\chi^{2}=2.87 ; \mathrm{df}=2\right.$; $\mathrm{P}=0.24)$. $\mathrm{P}$-values for both female horses and geldings were $<0.2$ and therefore included in the multivariable logistic regression models. No significant association between seropositivity and age groups was detected $\left(\chi^{2}=6.21, \mathrm{df}=5, \mathrm{P}=0.20\right)$. Horses aged between 21 and 25 years and those $\leq 5$ years old recorded a $\mathrm{P}$-value $<0.2$ and were included in the multivariable logistic regression models. Analysis of the seroprevalence of BFV and breed of the horses showed a significant association between seropositivity and breed $\left(\chi^{2}=11.56, \mathrm{df}=5, \mathrm{P}=0.04\right)$. Quarter Horses, Thoroughbreds, crossbred horses, ponies and 'other' breeds were all noted to have $\mathrm{P}$-values $<0.2$ and were therefore included in the multivariable logistic regression models. 
Table 1. Apparent prevalence and univariate results for Ross River virus by demographic, geographic and management factors

\begin{tabular}{|c|c|c|c|c|c|c|c|c|}
\hline \multirow[t]{2}{*}{ Variable } & \multirow[t]{2}{*}{ No. of horses tested } & \multicolumn{5}{|c|}{ No. of positive horses } & \multirow[t]{2}{*}{$\chi^{2}$} & \multirow[t]{2}{*}{ P value } \\
\hline & & Total & AP (\%) & OR & Lower 95\% Cl & Upper $95 \% \mathrm{Cl}$ & & \\
\hline \multicolumn{9}{|l|}{ Sex } \\
\hline Female $^{\mathrm{b}}$ & 127 & 115 & 90.55 & 0.84 & 0.37 & 1.96 & 0.17 & 0.342 \\
\hline Male & 1 & 1 & 100.00 & - & 0.01 & - & 0.10 & 0.457 \\
\hline Gelding $^{\mathrm{b}}$ & 160 & 145 & 90.63 & 0.82 & 0.34 & 1.90 & 0.22 & 0.326 \\
\hline \multicolumn{9}{|l|}{ Age (years) } \\
\hline$\leq 5^{b}$ & 39 & 35 & 89.74 & 0.81 & 0.28 & 2.89 & 0.14 & 0.342 \\
\hline $6-10$ & 96 & 89 & 92.71 & 1.31 & 0.54 & 3.49 & 0.35 & 0.287 \\
\hline $10-15$ & 71 & 62 & 87.32 & 0.55 & 0.23 & 1.36 & 1.90 & 0.933 \\
\hline $16-20$ & 54 & 50 & 92.60 & 1.23 & 0.43 & 4.36 & 0.14 & 0.376 \\
\hline $21-25^{b}$ & 22 & 21 & 95.45 & 2.08 & 0.36 & 45.24 & 0.51 & 0.267 \\
\hline$\geq 26$ & 6 & 6 & 100.00 & - & 0.14 & 0.14 & 0.58 & 0.289 \\
\hline \multicolumn{9}{|l|}{ Breed } \\
\hline Australian Stockhorse & 55 & 53 & 93.36 & 2.89 & 0.76 & 18.72 & 2.18 & 0.682 \\
\hline Quarter Horse ${ }^{\mathrm{b}}$ & 50 & 46 & 92.00 & 1.11 & 0.39 & 3.95 & 0.04 & 0.446 \\
\hline Thoroughbred $^{\mathrm{b}}$ & 80 & 71 & 88.55 & 0.66 & 0.28 & 1.63 & 0.92 & 0.174 \\
\hline Crossbred $^{\mathrm{b}}$ & 53 & 46 & 92.00 & 0.55 & 0.22 & 1.48 & 1.68 & 0.108 \\
\hline Pony ${ }^{b}$ & 20 & 19 & 95.00 & 1.87 & 0.32 & 10.78 & 0.37 & 0.308 \\
\hline Other $^{b}$ & 30 & 28 & 93.33 & 1.37 & 0.35 & 9.02 & 0.17 & 0.37 \\
\hline \multicolumn{9}{|l|}{ Average temperature $\left({ }^{\circ} \mathrm{C}\right)^{\mathrm{b}}$} \\
\hline$<23$ & 14 & 11 & 78.57 & 3.31 & 0.65 & 13.55 & 3.04 & 0.111 \\
\hline$\geq 23$ & 133 & 123 & 92.48 & & & & & \\
\hline \multicolumn{9}{|c|}{ Average annual rainfall $(\mathrm{mm})^{\mathrm{b}}$} \\
\hline$<1000$ & 40 & 38 & 95.00 & 2.17 & 0.51 & 15.00 & 1.01 & 0.171 \\
\hline$\geq 1000$ & 107 & 96 & 89.72 & & & & & \\
\hline \multicolumn{9}{|l|}{ Water sources in locality ${ }^{c}$} \\
\hline Yes & 76 & 70 & 92.10 & 1.27 & 0.39 & 4.24 & 0.18 & 0.344 \\
\hline No & 71 & 64 & 90.10 & & & & & \\
\hline \multicolumn{9}{|c|}{ Large numbers of mosquitoes on property ${ }^{b}$} \\
\hline Yes & 121 & 113 & 93.34 & 3.33 & 0.91 & 11.34 & 4.23 & 0.034 \\
\hline No & 26 & 21 & 80.77 & & & & & \\
\hline \multicolumn{9}{|c|}{ Other species of animals in locality } \\
\hline Avian $^{\mathrm{b}}$ & 15 & 15 & 100.00 & - & 0.44 & - & 1.62 & 0.116 \\
\hline Bovine $^{b}$ & 59 & 53 & 89.83 & 0.76 & 0.24 & 2.55 & 0.21 & 0.325 \\
\hline Canine $^{b}$ & 79 & 72 & 91.14 & 1.00 & 0.30 & 3.23 & 0.08 & 0.5 \\
\hline Marsupial & 25 & 23 & 92.00 & 1.14 & 0.26 & 8.01 & 0.03 & 0.463 \\
\hline Porcine ${ }^{b}$ & 24 & 21 & 87.50 & 0.62 & 0.16 & 3.01 & 0.48 & 0.249 \\
\hline
\end{tabular}

${ }^{\text {a }}$-value for $\chi^{2}$-test. ${ }^{b}$ Variable included in multivariable logistic regression model. ${ }^{c}$ Waterways, marshlands, coast, wetlands, creeks, rivers and/or dams. AP, apparent prevalence; $\mathrm{Cl}$, confidence interval; $\mathrm{OR}$, odds ratio.

No association could be shown between the seroprevalence of BFV on the horse properties and annual average temperature (Yates' $\chi^{2}=0.01, \mathrm{df}=1, \mathrm{P}=0.50$ ), amount of rainfall (Yates' $\chi^{2}=0.0, \mathrm{df}=1$, $\mathrm{P}=0.50$ ) or the location of the horse properties in close proximity to waterways, marshlands, coastal regions, wetlands, creeks, rivers and/or dams (Yates' $\chi^{2}=0.03$; $\mathrm{df}=1 ; \mathrm{P}=0.43$ ). Unlike RRV, large numbers of mosquitoes were not found to be significantly associated with seropositive horse properties $\left(\chi^{2}=0.45 ; \mathrm{df}=1\right.$; $\mathrm{P}=0.25)$ in the univariate analysis. The presence of other animal species, including avian, bovine, porcine, canine and marsupial, on the property and/or on those within the neighbourhood, and their association to BFV were also analysed. The presence of avian, bovine, canine and porcine species (domestic and wild) were all found to have $\mathrm{P}$-values $<0.2$ in the univariate analysis and were analysed further within the multivariable logistic regression models. Avian and porcine species were found to be the only remaining significant associations with BFV seropositivity (Table 4). 
Table 2. Final multivariable logistic regression models for Ross River virus seropositive properties

\begin{tabular}{lccrrrr}
\hline Independent variable & Regression coefficient & SE & Wald Z-value & Wald P-value & $\begin{array}{c}\text { Lower } \\
95 \% \mathrm{Cl}\end{array}$ & $\begin{array}{c}\text { Upper } \\
95 \% \mathrm{Cl}\end{array}$ \\
& & & & & $\begin{array}{c}\text { OR } \\
9\end{array}$ \\
\hline B0: intercept & -0.85 & 0.40 & -2.121 & 0.03391 & -1.64 & -0.07 \\
B1: (mosquitoes $=1$ ) & 1.16 & 0.62 & 1.879 & 0.06022 & -0.05 & 2.36 \\
\hline
\end{tabular}

$\mathrm{Cl}$, confidence interval; OR, odds ratio; $\mathrm{SE}$, standard error.

\section{Whataroa virus}

Previous exposure to Whataroa-like virus was detected in one horse in the Far North Coast-Tableland region and one donkey in the Mackay-Whitsunday region (3.6\%; 95\% CI $-3.3-10.45 \%$ and $3.3 \%$; 95\% CI $-3.3-9.76 \%$, respectively). This was the only donkey bled in the study and it was included at the request of the owner. The proportion of properties found to be seropositive to WHAV was $1.36 \%$ (95\% CI $-0.51-3.23 \%$ ). Because of the low prevalence and the high probability of false-positive results, analysis of risk factors associated with prevalence of WHAV was not conducted.

\section{Discussion}

Because of the clustering of horses on properties, seroprevalence was analysed and is reported in terms of the sampling unit 'property' and not by each individual horse. Although the data suggested that the overall seroprevalence of RRV in the horse population of northern Queensland was $91 \%$, BFV $15 \%$ and WAV almost 1.5\%, these data should be interpreted with caution when extrapolated to the general population because of the study having to rely largely on convenience sampling, which may not be truly representative of the entire northern Queensland horse population. The results do, however, show that RRV is endemic at a very high prevalence along the east coast of northern Queensland. Because relatively few horses were sampled on each property, it is likely that the proportion of properties that have RRV may be higher than the results suggest.

The antibody titres of the horses seropositive to RRV ranged from 10 to 1280 , with the most frequently recorded titre being 80 for $29 \%$ of serum samples. Only $6 \%$ of the sampled horses were noted to have titres $\geq 640$. These findings, in conjunction with the lack of ownerreported clinical signs associated with RRV, suggested that the virus has a low virulence and does not cause significant clinical disease and/or horses in the region are exposed at an early age and have constant challenge resulting in a non-susceptible population that has persistent immunity.

Sample analysis revealed an association between seropositivity of RRV and horse properties with large numbers of mosquitoes. However, no significant association between seropositivity and degree of rainfall or the location of the horse properties in close proximity to waterways, marshlands, coastal regions, wetlands, creeks, rivers and/or dams was found. This can probably be explained by the fact that very few properties did not have water sources, making it statistically difficult to show an association with the presence of water on properties. Studies in more arid regions with fewer water reservoirs may reveal different associations related to mosquito habitat.

Across eastern Australia, sampling of macropod populations has revealed a seroprevalence of RRV as high as $89 \% .{ }^{16}$ This evidence suggests an ecologically significant association of large marsupials, especially wallabies, with RRV and that the basic epidemiology of the human disease epidemic polyarthritis may therefore involve cycles of transmission between these large macropods and mosquitoes. ${ }^{16}$ In the present study, no association could be shown in the multivariable logistic regression analysis to the presence of other animal species, including avian, bovine, porcine, canine and marsupial, on the property and/or on those within the neighbourhood. This could be because almost all properties sampled were found to be in contact with each of the aforementioned species and consequently may have influenced the outcome of this analysis. However, in the univariate analysis there seemed to be some association with the presence of birds and pigs on properties, which may warrant further investigation.

Results of experimental infection of non-human vertebrates with RRV has identified maximum titres of viraemia, as expressed as the minimum dose sufficient to kill $50 \%$ of inoculated mice (LD50) per gram of undiluted blood. ${ }^{17,18}$ It is interesting to note that horses have one of the highest levels of induced viraemia (6.3 $\log _{10} / \mathrm{LD}_{50}$ ), similar to that of the agile wallaby (Macropus agilis: $\left.7.4 \log _{10} / \mathrm{LD}_{50}\right)$ and the gray kangaroo $\left(M\right.$. giganteus: $\left.6.1 \log _{10} / \mathrm{LD}_{50}\right){ }^{17}$ The highest maximum titre of viraemia has been recorded from the marsupial mouse (Antechinus sp.: $7.4 \log _{10} / \mathrm{LD}_{50}$ ) and the lowest from the little corella (Cacatua sanguinea: $<2.3 \log _{10} / \mathrm{LD}_{50}$ ). ${ }^{17,18}$ The question then is whether horses can act as amplifier hosts based on the previously reported high levels of viraemia possible and the high seroprevalence reported in the current study. Outbreaks of RRV in humans in major metropolitan centres, where there are not significant numbers of macropods, have also led to speculation that horses may act as amplifying hosts. ${ }^{19}$ It is known that horses can experimentally infect Culex annulirostris (a major vector of RRV) and that under experimental conditions viraemia may last up to 5 days in horses. Hence, in peri-urban areas the literature indicates that horses may play a role as amplifying hosts when present in appreciable numbers, before seroconversion and clinical signs, providing there exists a competent host-vector relationship.

Many of the horses bled in this study were hobby horses and would have come from peri-urban small holdings where there is often an abundance of mosquitoes. Azuolas et al. ${ }^{2}$ point out that 
Table 3. Apparent prevalence and univariate results for Barmah Forest virus by demographic, geographic and management factors

\begin{tabular}{|c|c|c|c|c|c|c|c|c|}
\hline \multirow[t]{2}{*}{ Variable } & \multirow[t]{2}{*}{ No. of horses tested } & \multicolumn{5}{|c|}{ No. of positive horses } & \multirow[t]{2}{*}{$\chi^{2}$} & \multirow[t]{2}{*}{ P value ${ }^{4}$} \\
\hline & & Total & AP (\%) & OR & Lower $95 \% \mathrm{Cl}$ & Upper 95\% Cl & & \\
\hline \multicolumn{9}{|l|}{ Sex } \\
\hline Female $^{\mathrm{b}}$ & 127 & 6 & 4.72 & 0.45 & 0.16 & 1.16 & 2.74 & 0.051 \\
\hline Male & 1 & 0 & 100.00 & 0.00 & 0.00 & 229.70 & 0.08 & 0.462 \\
\hline Gelding $^{b}$ & 160 & 16 & 10.00 & 2.25 & 0.87 & 6.45 & 2.85 & 0.048 \\
\hline \multicolumn{9}{|l|}{ Age (years) } \\
\hline$\leq 5^{b}$ & 39 & 1 & 2.56 & 0.29 & 0.01 & 1.62 & 1.65 & 0.103 \\
\hline $6-10$ & 96 & 9 & 9.38 & 1.42 & 0.56 & 3.48 & 0.62 & 0.22 \\
\hline $11-15$ & 71 & 4 & 6.00 & 0.66 & 0.19 & 1.92 & 0.54 & 0.245 \\
\hline $16-20$ & 54 & 4 & 7.40 & 0.96 & 0.27 & 2.81 & 0.01 & 0.491 \\
\hline $21-25^{\mathrm{b}}$ & 22 & 4 & 18.18 & 3.04 & 0.81 & 9.60 & 3.75 & 0.046 \\
\hline$\geq 26$ & 6 & 0 & - & 0.00 & 0.00 & 8.07 & 0.51 & 0.309 \\
\hline \multicolumn{9}{|l|}{ Breed } \\
\hline Australian Stockhorse & 55 & 4 & 7.27 & 0.94 & 0.26 & 2.74 & 0.01 & 0.475 \\
\hline Quarter Horse $^{\mathrm{b}}$ & 50 & 7 & 14.00 & 2.41 & 0.87 & 6.21 & 3.47 & 0.043 \\
\hline Thoroughbred $^{\mathrm{b}}$ & 80 & 1 & 1.25 & 0.11 & 0.01 & 0.63 & 6.41 & 0.003 \\
\hline Crossbred $^{\mathrm{b}}$ & 53 & 6 & 11.32 & 1.74 & 0.60 & 4.60 & 1.25 & 0.142 \\
\hline Pony ${ }^{b}$ & 20 & 0 & - & 0.00 & 0.00 & 1.94 & 1.78 & 0.096 \\
\hline Other $^{b}$ & 30 & 4 & 13.33 & 2.05 & 0.56 & 6.22 & 1.54 & 0.124 \\
\hline \multicolumn{9}{|l|}{ Average temperature $\left({ }^{\circ} \mathrm{C}\right)^{\mathrm{b}}$} \\
\hline$<23$ & 14 & 2 & 14.29 & 0.94 & 0.13 & 4.10 & 0.01 & 0.498 \\
\hline$\geq 23$ & 133 & 20 & 15.04 & & & & & \\
\hline \multicolumn{9}{|l|}{ Av. annual rainfall $(\mathrm{mm})^{\mathrm{b}}$} \\
\hline$<1000$ & 40 & 6 & 15.00 & 1.00 & 0.34 & 2.99 & 0.00 & 0.487 \\
\hline$\geq 1000$ & 107 & 16 & 14.95 & & & & & \\
\hline \multicolumn{9}{|l|}{ Water sources in locality ${ }^{c}$} \\
\hline Yes & 76 & 11 & 14.50 & 0.93 & 0.37 & 2.33 & 0.03 & 0.433 \\
\hline No & 71 & 11 & 15.50 & & & & & \\
\hline \multicolumn{9}{|c|}{ Large numbers of mosquitoes on property ${ }^{b}$} \\
\hline Yes & 121 & 17 & 14.05 & 0.68 & 0.23 & 2.29 & 0.45 & 0.253 \\
\hline No & 26 & 5 & 19.23 & & & & & \\
\hline \multicolumn{9}{|c|}{ Other species of animals in locality } \\
\hline Avian $^{\mathrm{b}}$ & 15 & 6 & 40.00 & 4.76 & 1.41 & 15.43 & 8.23 & 0.007 \\
\hline Bovine $^{b}$ & 59 & 12 & 20.34 & 1.98 & 0.78 & 5.09 & 2.24 & 0.074 \\
\hline Canine $^{\mathrm{b}}$ & 79 & 16 & 20.25 & 2.61 & 0.98 & 7.70 & 3.75 & 0.028 \\
\hline Marsupial & 25 & 5 & 20.00 & 1.54 & 0.46 & 4.55 & 0.60 & 0.224 \\
\hline Porcine ${ }^{b}$ & 24 & 9 & 37.50 & 5.00 & 1.78 & 13.91 & 11.44 & 0.001 \\
\hline
\end{tabular}

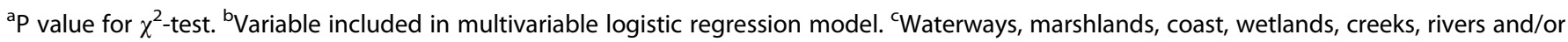
dams. AP, apparent prevalence; $\mathrm{Cl}$, confidence interval; OR, odds ratio.

in their studies, RRV isolation was only achieved from a small percentage of horses with RRV IgM titres and not from horses with RRV IgG titres. Hence the window for viraemia appears to be fairly limited in horses if they do act as amplifiers. The role of horses as potential amplifying hosts in both enzootic and epizootic virus transmission cycles of RRV remains uncertain under extensive pastoral conditions where horse population densities may be low.
Similar to RRV, the highest rates of BFV infection have been reported in the northern tropics: Northern Territory (37 per $100,000)$ and Queensland (21.5 per 100,000), with the largest overall number of BFV infection notifications recorded in Queensland. ${ }^{1}$ Additionally, coastal areas of the state have recorded a higher occurrence of BFV disease than further inland regions. ${ }^{20}$ As well as affecting human populations, seropositivity to BFV has been identified in horses; however, prevalence in Australia's equine population has not 
Table 4. Final multivariable logistic regression model for Barmah Forest virus seropositive properties

\begin{tabular}{lccccrrr}
\hline Independent variable & Regression coefficient & SE & Wald Z-value & Wald P-value & Lower 95\% Cl & Upper 95\% Cl & OR \\
\hline B0: intercept & -0.44 & 0.19 & -2.33 & 0.02 & -0.82 & -0.07 \\
B1: (porcine = 1) & 1.43 & 0.56 & 2.54 & 0.01 & 0.64 \\
B2: (avian = 1) & 1.26 & 0.64 & 1.96 & 0.05 & 0.33 & 2.53 \\
\hline
\end{tabular}

$\mathrm{Cl}$, confidence interval; $\mathrm{OR}$, odds ratio; $\mathrm{SE}$, standard error.

yet been investigated. ${ }^{6}$ Within the present study, overall seroprevalence of $7.6 \%$ was measured and no significant difference observed between the three regions of northern Queensland. If clinical disease associated with BFV is suspected to be similar to that of RRV in horses, testing of horses displaying associated clinical signs should be considered in northern Queensland. This may identify horses previously unknown to have been infected by BFV and assist in qualifying the signs and severity, if any, of disease associated with this virus.

BFV is considered to share similar hosts to RRV. The significance of the association between those properties in close proximity to avian and porcine species (domestic and wild) and seroprevalence of BFV is unknown. Further studies into the seroprevalence of BFV in avian and porcine species would be required to determine the significance of BFV within these species.

WHAV has not been isolated from any source in Australia other than mosquitoes captured in New South Wales. ${ }^{7}$ Information on the ecology and epidemiology of the virus in Australia is lacking. WHAV M78 (the specific New Zealand strain) is phylogenetically and antigenically very closely related to other known WHAV-like virus isolates from New South Wales, South Australia and Western Australia, and also to the Sindbis-like type-2 Australian virus isolates in the Western equine encephalitis antigenic complex of alphaviruses. ${ }^{21}$ Sinbis virus is a relatively common alphavirus in Australia and hence the equine seroconversions evidenced in this study could therefore very plausibly be cross-reactions not specifically to WHAV, but to other WHAV-like and/or Sindbis-like viral strain/s circulating in northern Queensland. ${ }^{7}$ Although the data were suggestive of WHAV being prevalent in the northern Queensland horse population, more comprehensive investigations into the status of the virus and related viruses in Queensland are required.

\section{Conclusions}

The current findings of a large number of horses within the state being seropositive to RRV indicated the disease is endemic within the horse population and that a high proportion of horses may have immunity, which could explain why horses infected by RRV in this region do not demonstrate significant clinical signs. The role of horses as amplifier hosts should also be considered. Exposure to BFV appears to be significant in northern Queensland and this should be considered as a differential diagnosis for RRV for horses in these tropical regions. The role of WHAV remains uncertain. Further studies on all these viruses would be extremely beneficial from a public health perspective and would ultimately enable more comprehensive data on the epidemiology of all three viruses to be obtained.

\section{Acknowledgments}

The authors would like to acknowledge the many veterinarians, horse owners and clubs of northern Queensland who willingly participated in this study, Michelle Gummow for assisting with the figures and James Cook University for funding this work.

\section{Conflicts of interest and sources of funding}

The authors declare no conflicts of interest or external sources of funding for the work presented here.

\section{References}

1. Corvisy R, Trungrove M, Bright A et al. Australia's notifiable disease status, 2012: Annual Report of the National Notifiable Diseases Surveillance System. Commun Dis Intell Q Rep 2015:E46-E136.

2. Azuolas J, Wishart E, Bibby $S$ et al. Isolation of Ross River virus from mosquitoes and from horses with signs of musculoskeletal disease. Aust Vet $J$ 2003;81:344-347.

3. Cloonan M, O'Neill B, Vale T et al. Ross River virus activity along the south coast of New South Wales. Aust J Exp Biol Med Sci 1982;60:701-706.

4. Liu C, Begg K, Johansen C et al. Communicable Diseases Network Australia National Arbovirus and Malaria Advisory Committee Annual Report, 2006-07. Commun Dis Intell Q Rep 2008;32:31-47.

5. Phillips D, Murray J, Aaskov J et al. Clinical and subclinical Barmah Forest virus infection in Queensland. Med J Aust 1990;152:463-466.

6. Kay B, Boyd A, Ryan P et al. Mosquito feeding patterns and natural infection of vertebrates with Ross River and Barmah Forest viruses in Brisbane, Australia. Am J Trop Med Hyg 2007;76:417-423.

7. Saleh S, Poidinger M, Mackenzie J et al. Antigenic and genetic typing of Whataroa viruses in Australia. Am J Trop Med Hyg 2004;71:262-267.

8. Elizabeth Macarthur Agricultural Institute laboratory. Serum microneutralisation test. EMAI, Menangle, NSW, 2015.

9. Humphry R, Cameron A, Gunn G. A practical approach to calculate sample size for herd prevalence surveys. Prev Vet Med 2004;65:173-188.

10. Thrusfield M. Veterinary epidemiology. 3rd edn. John Wiley \& Sons, London, 2007. 11. Reed L, Muench H. A simple method of estimating fifty per cent endpoints. Am J Epidemiol 1938;27:493-497.

12. Cameron A. Survey toolbox: a practical manual and software package for active surveillance of livestock diseases in developing countries. Australian Centre for International Agricultural Research, Canberra, ACT, 1999.

13. Katz MH. Multivariable analysis: a pratical guide for clinicians. Cambridge University Press, Cambridge, UK, 1999.

14. Hintze J. NCSS statistical software. 9th edn. NCSS Statistical Software, Kaysville, UT, USA, 2013. www.ncss.com

15. Armitage P, Berry G. Statistical methods in medical research. 3rd edn. Blackwell Scientific Publications, Oxford, UK, 1994.

16. Doherty $R$, Standfast $H$, Domrow $R$ et al. Studies of the epidemiology of arthropod-borne virus infections at Mitchell River Mission, Cape York Peninsula, North Queensland. IV: arbovirus infections of mosquitoes and mammals, 1967-1969. Trans R Soc Trop Med Hyg 1971;65:504-513. 
17. Kay B, Aaskov J. Ross River virus (epidemic polyarthritis) CRC Press, Boca Raton, FL, USA, 1989.

18. Whitehead R. Experimental infection of vertebrates with Ross River and Sindbis viruses, two group A arboviruses isolated in Australia. Aust J Exp Biol Med Sci 1969;47:11-15.

19. Mackenzie JS, Broom AK, Hall RA et al. Arboviruses in the Australian region 1990 to 1998. Commun Dis Intell 1998;22:93-100.
20. Naish S, Mengerson K, Hu W et al. Wetlands, climate zones and Barmah Forest virus disease in Queensland, Australia. Trans $R$ Soc Trop Med Hyg 2012;106:749-755.

21. Centers for Disease Control and Prevention. The International Catalog of Arboviruses. CDCP, 2017. https://wwwn.cdc.gov/arbocat/. Accessed October 2017. 